\title{
Conception and Evaluation of Internet of Things Connecting to RFID Framework
}

\author{
${ }^{1}$ M. El Harrak Hajri, ${ }^{1}$ A Asselman, ${ }^{2}$ M. Yassin Chkouri \\ ${ }^{1}$ Physic Departement, Science Faculty of UAE, Tetouan, Morroco \\ ${ }^{2}$ Informatic Departement, ENSA Tetouan of UAE, Tetouan, Morroco, \\ harrakmed@gmail.com, adelasselman@gmail.com, yassin.chkouri@gmail.com
}

\begin{abstract}
The notion of the Internet of the Objects designates a network of objects connected to each other and communicating automatically. In this paper we develop the notions of semantics are becoming increasingly important because, more than ever, they appear as a solution to problems of interoperability and interpretation of data and services by machines. The diversity of possible applications at the intersection of the Internet of Things and the Semantic Web has prompted many research teams to work at the interface between these two disciplines. We wish in this paper to make an inventory of their proposals. We also seek to contribute to the evolution of this field of research by proposing an ontology to describe networks of connected objects. Keywords: Ontologies, Internet of Objects, Interoperability, Data Enrichment.
\end{abstract}

Keywords-RFID ;Internet of things ; network topology ; microcontrolles

\section{Introduction}

In recent years, the Internet of objects (IoT in English 1) has evolved at an exceptional speed, connecting a large number of heterogeneous objects (sensors, actuators, smartphones, applications, etc.). The loT contributes significantly to the production of data under Big Data, with an estimate of 4.4 billion GB produced in 2013, with some estimates projecting the existence of more than 26 billion loT devices connected to the " Horizon 2020 2. One of the main concepts of the internet of objects is machine-tomachine (M2M). M2M is the association of information and communication technologies, with communicating machines in order to provide the latter with the means to interact without human intervention. The fields of application are thus very wide: energy management, home automation, medical diagnostic aid, comfort of life, etc. M2M has suffered from a vertical fragmentation of the approaches adopted to cover the needs of the different fields of application. To solve this problem, in 2012 the European Standardization Institute (ETSI) developed a standard specification for a horizontal M2M service platform, the first of which was provided by LAAS. This makes it possible to collect data and manage various objects in a transparent way. The challenge now lies in the use of these data. The best approach seems to be to associate semantics to these data through ontologies in order to facilitate their reuse and to allow the implementation of the reasoning mechanisms. Recent works aim to propose ontologies allowing to represent the data collected by connected objects as well as the interactions between these objects Barnaghi et al. (2012), L. Atzori \& Morabito (2010). These approaches are, for the 
most part, specific to the types of objects for which they are designed and to the data they collect. A real challenge is to define a generic approach for the semantization of data with a dual objective: to promote interoperability between objects, and to make data usable automatically. They could then be integrated into platforms that implement automatic diagnostic and supervisory mechanisms, which is crucial for $\mathrm{M} 2 \mathrm{M}$. The purpose of this paper is twofold. We first present a panorama of the needs that ontologies can meet in the field of IoT and present the ontologies currently available in the field. On the basis of this analysis, we propose an ontology to capitalize on the one hand the work done in the field and on the other hand to take into account the mature advances in the field of Knowledge Engineering. The paper is organized in the following way: Section 2 presents a state of the art in the use of ontologies in loT, Section 3 describes the loT-O ontology that we propose and Section 4 illustrates its interest From a real case[1].

\section{Concepts and Definitions}

\subsection{The Internet of Things}

The internet of objects is a technological revolution in the field of computing and telecommunications [2, 3]. IoT refers to a variety of detection equipment and information systems such as sensor arrays, reading devices (RFID, barcode), short-range location and communication systems based on Machine-to-machine (M2M) communication, through the Internet to form a larger and smarter network [4]. This revolution is based on a constant evolution of the Internet, technologies and software, communication protocols, embedded sensors that are constantly being improved, physical objects that are increasingly intelligent and capable of providing information and To perceive their environment in real time [8]. The loT can be seen from two angles, either centered on the Internet or centered on the object. When it is centered on the Internet, services are the main component of its architecture and the objects contribute by feeding it with data. When centered on the object, the center of the architecture becomes the object and one speaks of the cloud of the objects. The cloud of objects thus appears as a platform of objects allowing intelligent use of infrastructures, applications and information at reduced costs [5].

IoT is based on a wide range of technologies, protocols, networks and concepts: network infrastructures, new software, hardware and services platforms. In particular, loT is associated with identification and traceability via the integration of RFID (Radio Frequency Identification Systems) chips; The semantic web, nanotechnologies, mobility, ubiquity, crowdfunding [6]. This constant and evolving change in technologies and the advent of new platforms, new services and new architectures bring new prospects, new markets with wide, varied economic, social, political, ethical, security and regulatory challenges.

We can cite as challenges the integration and sharing of data on Cloud platforms, securing personal data of users (freedom and confidentiality), good governance (transparent and democratic), harmonization of standards, networks And the vagaries of economic competition [7]. The management of the healthcare chain is in this perspective and occupies a preponderant place among the fields of application of these new technologies and concepts related to the loT. We can cite as examples the problems dealt with in the field of healthcare, as discussed in [8], such as management and decision support, stock optimization, Quality of service, radio frequency identification, real-time tracking of products and processes.

\subsection{The health chain}

Sanitation can be seen as the integration and collaboration of a set of activities whose purpose is to plan, implement and control a flow of materials, semi-finished objects and finished projects, Origin at point of 
M. El Harrak Hajri, A Asselman, M. Yassin Chkouri. Conception and Evaluation of Internet of Things Connecting to RFID Framework. Transactions on Machine Learning and Artificial Intelligence, Vol 5 No August (2017); p: 572-583

use. These activities include planning and forecasting, control and storage, handling, order processing and distribution, marketing, purchasing, packaging, after-sales service [9]. All these activities result in productrelated information flows and financial flows, the optimization of which is a key factor for the competitiveness of companies. Controlling flows (physical flow and information flow) makes it possible to make strategic and tactical decisions for the good governance of the company according to the specific constraints that may be financial (inventory immobilization costs, outstandings), physical (Warehouse location) or environmental (reduction of pollution, waste and energy consumption) [10].

Many tools and software have been created to facilitate the control of these flows. These tools include Enterprise Resource Planning (ERP), Advanced Planning System (APS), WareHouse Management System (WMS), Transport Management System (TMS), Customer Relationship Management (CRM) [11].

Most of these tools are struggling to adapt to the new challenges of the current health chain, namely demand uncertainty, coordination and global governance of the health chain [12], An intelligent and dynamic control [13], the flexibility of the chain to better meet the demands of the customer [14]. Similarly, these tools must deal with risk management and local and global decision-making for a decentralized health chain [15]; The interoperability of health networks with the constraints of heterogeneity of norms and standards. The management and exploitation of the flow of information generated by networks and healthcare platforms as an added value for the competitiveness of companies and as a vector for the creation of new structures and markets ("virtual enterprises", "enterprise 2.0" ) Represent challenges to be addressed by the aforementioned tools [16]. The Internet of objects as presented above contributes to the resolution of some of these great challenges. We cite as an example the identification by radio frequencies of products and sanitary objects (RFID, NFC (Near Fields Communication)) whose uses are multiple: traceability of products, management of the production chain, management of subscribers in Transport and leisure,

\section{The Internet Technologies of Things}

The Internet of objects aims to connect objects to each other via Internet protocols. The THING here represents everything around us (machines, mobile phones, computers, sensors) [17]. To achieve this goal, it is imperative to be able to identify the objects, assigning them a virtual interface so that they can communicate with their environment. It is important to note that more than six billion objects will be connected by 2015 [18]. The technological domains covered by the loT are broad and varied, as illustrated by the deployment diagram in Figure 3.

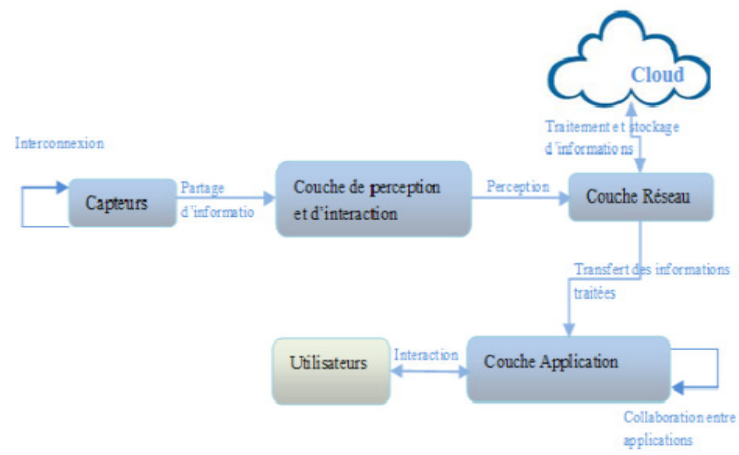

Figure 1: Deployment diagram in the loT [19]. 
In the following, we present a non-exhaustive list of the different lo concepts and technologies and their applications in the field of health.

\subsection{Architectures, Protocols and Algorithms}

New models of architecture make it possible to integrate sensors and the Internet. This communication between a sensor and the Cloud is done by a virtual layer that implements the operation of the actual sensors. Such a layer gives rise to new architectures called sensor arrays (Sensor Cloud) or Cloud of virtual sensors [20]. With such an architecture it is possible to create services based on virtual sensors, that is to say geographically distributed sensor environments which can be used on demand by several users.

In the field of sanitation, and in particular in the management of wind farms, such architectures are used for the transmission of data for preventive maintenance purposes. In this area, many other applications are underway to link mathematical optimization algorithms to web services to better harness the data collected by the sensors in order to provide better tools and maintenance services [19]. With the loT, billions of objects will be connected to the Internet, each object possibly possessing its own sensors. This ecosystem poses problems in identifying and locating objects and services (sensor, actuators, pallets, container, service, etc.) uniquely in the Internet, and makes it even more difficult to manage the enormous volume Of information generated by these sensor networks.

To address this issue, several protocols and algorithms have emerged, including CASSARAM, a middleware offering semantic-based discovery models; The Objects Naming Service (ONS) to address

In order to facilitate the search and the identification of the objects, to improve the communication between the objects and the platforms of the Cloud. The ONS is a naming service that distributes information about the source of a product, from the manufacturer to the consumer. It is based on the same principle as the DNS (Domain Name System), to access it simply by knowing the product identifier EPC (Electronic Product Code), or the GTIN (Global Trade Item Number) [12]. Several architectures (2Tiers, 3-Tiers) linking the production chain to the loT have been proposed in order to set up manufacturing platforms based on Cloud Computing [15]. Tools for driving the production line from RFID tags, as is the range of IFM products used in the production chain. An application of these new architectures based on the $I O$ in healthcare is the production chain where it is a question of sharing the resources (machines, robots) and the production capacities optimally, and make the resource allocation to the [17]. Another feature of this application is the ability to use these protocols to inform the customer about the origin and content of the product. By integrating sensor data with Cloud platforms, loT architectures provide wider use possibilities that extend beyond the sanitary sphere.

\subsection{Interfacing technologies (RFID, NFC, Zigbee) in the IoT}

Several technologies are used to communicate an object with the Internet, including RFID, NFC, Zigbee communication protocol [19]. Other solutions are being developed such as acoustic identification systems, microwaves, optical systems, the use of DNA, software marking or the integration of chips in the design of objects [18]. The RFID consists of a read / tag pair. The reader sends a radio wave, the label in turn sends an identification frame. Once the chip is fed, the tag and tag communicate according to the Tag Talk First (ITF) or ITF (Interogator Talk First) protocol. In TTF mode, the tag first transmits the information contained in the chip to the interrogator. In ITF mode, the interrogator sends a request to the tag, and the tag responds later. There are three types of labels, passive, active and semi-active labels. The former do not have their own source of energy: a small amount of energy is provided by the magnetic field 
M. El Harrak Hajri, A Asselman, M. Yassin Chkouri. Conception and Evaluation of Internet of Things Connecting to RFID Framework. Transactions on Machine Learning and Artificial Intelligence, Vol 5 No August (2017); p: 572-583

induced by the reader at the time of identification. The active tags are battery-powered, they are capable of sending themselves identification information without soliciting a reader. The semi-active uses a hybrid mechanism: self-powered, they activate only at the request of the reader, allowing a lower energy consumption than the active tags. The reading distance of the RFID chips varies from a few $\mathrm{cm}$ to a few meters $(10 \mathrm{~m})$, and can go beyond $(200 \mathrm{~m})$ with long-range communication technologies [19]. The operating principle of the RFID technology is illustrated in Figure 5.

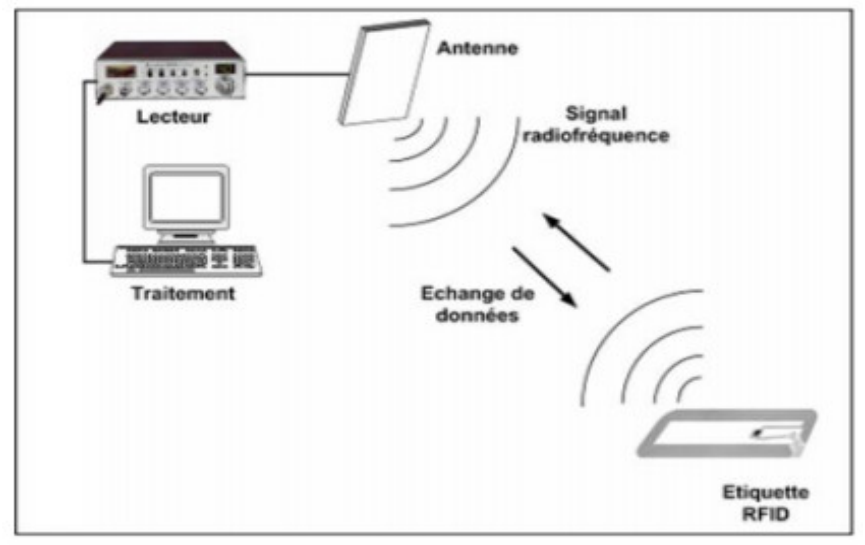

Figure 2: Principle of operation of RFID

NFC technology is the result of several evolutions of microcontrollers, smart cards, and short-range communications [11]. NFC is based on the same principle as RFID, ie identification by radio frequency. It allows the exchange of information at short distance between two objects (a reader and a card) without contact, and operates in two modes: passive mode and active mode. In passive mode, the user's terminal emulates a smart card and acquires energy from the reader's radiation (mobile phone, for example). In active mode, the terminal behaves like an electronic label reader (barcode, 2D labels) and has its own power source [20] (an on-board battery for example). NFC allows the user to exchange information with his environment, in particular in the field of transport, leisure, shopping, or reading information on billboards. The use of NFC facilitates the management of sales data in the health chain, management and validation of bus tickets in urban transport [11]. When a user scans an NFC tag, they can also access information about the product (origin, manufacturer, content / ingredients, manufacturing process) [11].

Zigbee is a low-cost wireless communication protocol that allows short-distance exchanges between nodes on a Wireless Personal Area Networks (WPAN) network. This protocol is based on the IEEE 802.15.4 standard, which specifies the communication protocols between the physical layers and the data link of the OSI model, by defining three types of equipment: Full Function Devices (FFDs) Complete, reduced Function Devices (RFDs), and network coordinators. The FFDs coordinate the entire network, they are Personal Area Network (PAN) coordinators, router or device connected to a sensor. RFDs are designed for simple applications such as lighting a lamp. RFDs can communicate only with an FFD [49]. Among the advantages of this communication protocol, we can mention the low energy consumption, the optimum use of the bandwidth, and its low implementation cost. These advantages make it possible to adopt the Zigbee protocol in embedded environments and industrial networks, or the development of new products based on this protocol [12]. 


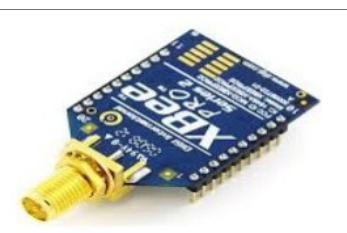

Figure 3: XBee Communication Module

XBee can be used to transmit data between sanitary objects (sensors on containers, RFID tags on products) and cloud environments (data processing, Big Data, Web services).

\subsection{Communication networks (GSM, UMTS, LTE / LTE-A, WSN, VANET) in the IoT}

With the advent of the loT, another challenge is the availability of reliable communication networks, both in terms of infrastructure and communication protocols. This challenge is linked to, among other things, the mobility of objects, the heterogeneity of data and platforms, access to information from any place, anytime and anywhere Device (PDA, Smartphone, tablet), which makes it more difficult to standardize protocols and algorithms. Moreover, the volume of data to be transmitted by the sensors places great stress on the availability of the bandwidth, hence the need to implement networks adapted to these new constraints. In the literature, mention is made of two approaches, the use of short-range wireless networks (Zigbee, WiFi) to connect objects to the Internet via a gateway; And conventional broadband cellular networks (4G, 3G). The use of Ultra Narrow Band (UNB) networks is booming because most objects do not require the bandwidth available in broadband networks, but rather Networks with very low costs and very low energy consumption. Among the players in these ultra-low-band networks, we can cite Sigfox, Neul and On-Ramp. Sigfox networks are characterized by their hierarchical structure; Servers that verify all data and route messages to information systems, UNB modems that communicate with base stations or cells to cover wide areas, and base stations that route messages to servers. Other solutions exist to address communication problems in 10 , namely the integration of ubiquitous wireless networks to manage object connectivity [18], virtualization of network resources (physical and virtual) to facilitate Sharing and availability of resources, Xbee networks [14], the use of wireless sensors, for home automation applications, environmental monitoring, and portal security. These technologies are used in urban transport sanitation for vehicle collision avoidance [15], the location of people in risk areas by the use of wireless sensors, the prediction of transport time for goods, Or in the medical facility to collect information during an organ transport [16].

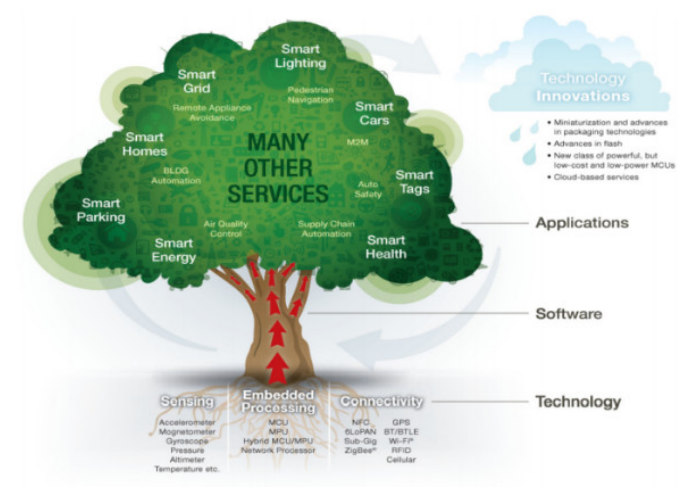

Figure4: the IOT - Different Services,Technologie, Meaning for everyone. 
M. El Harrak Hajri, A Asselman, M. Yassin Chkouri. Conception and Evaluation of Internet of Things Connecting to RFID Framework. Transactions on Machine Learning and Artificial Intelligence, Vol 5 No August (2017); p: 572-583

\section{Applications to Health}

This part of the paper divides applications of loT technologies into the four-point health chain, extracting and processing data for the purpose of steering the health chain, safeguarding this data which can be private and Privacy, marking and object detection technologies, and finally how to evaluate the performance of a system in the Internet of Things.

\subsection{Data extraction and processing in IOI}

"Big Data" is gradually and invasively replacing relational databases that have existed since the 1990s. These giant databases allow for the management of more data, their disparity and heterogeneity, Realtime access to information whether distributed or centralized. With the rise of the Internet of objects, sensors embedded on mobile devices make it possible to trace more and more data (temperature, pressure, GPS position, speed, luminosity, heart rate, etc.). This situation complicates more and more the task of analysts and data mining who are faced with the problem of proposing new solutions adapted to this steadily increasing volumetry, replacing the traditional searches that are based on General on statistical models, linear regression or health [20]. Also, it should be noted that the collection, formatting and transmission of data from sensors to Cloud platforms requires a lot of energy, which can be very constraining, so that we must now consider moving tasks Of processing to devices whose energy constraints are lower than those of the sensor (Smartphone, PDA, computer). There are middleware that have been developed in this sense (MOSDEN: Mobile Sensor Data Processing Engine) [18].

On the other hand, protocols that have been used for a few years for web applications (SOAP: REST: Representational State Transfer) are also used for object exchanges.

Data extraction languages (SPARC QL, ETL: Extract Transform and Load) facilitate the extraction and processing of data from various sources, distributed or non-distributed databases, relational databases or NoSQL. These languages, standards and protocols also aim to integrate the Internet of Things with Cloud Computing to give birth to the Cloud of Things [20], in order to create more intelligent applications, to make the data collected On objects that are more accessible and, above all, more relevant and meaningful.

The applications are diverse and varied in the field of healthcare, from production to management of relationships between all stakeholders in the healthcare chain including the client. At the production level, sensors (RFID tags) are integrated on products (pallets, bags, bins) to store information about their contents, traceability information (operations performed on products, origin of the product and its components , ...). Intelligent production line applications have emerged via RFID readers mounted on conveyors to automatically route items or pallets according to the operations to be performed or their contents.

On the other hand, it is possible to integrate the production chain and the Enterprise Resource Planning (ERM) systems, so that the data transmitted By intelligent sensors in the production chain can be used for local and global decision making for product manufacturing [18]. This integration also allows the strategic and tactical management of the company at global level (planning, management, production scheduling, resource management), and serves to optimize the maintenance of equipment in all areas (electric vehicle, wind, solar , Aeronautics, transport, ...). An example of an application is that of BMW which uses Win River technology to connect its on-board computer to telecommunication operators in order to dialogue in real time with cloud services (weather, traffic) for vehicle maintenance in case of breakdown. 
This service also makes it possible to retrieve information on the condition of important vehicle parts using integrated sensors, as well as statistics (information on the state of the road, flow of traffic) and More accurate diagnostics.

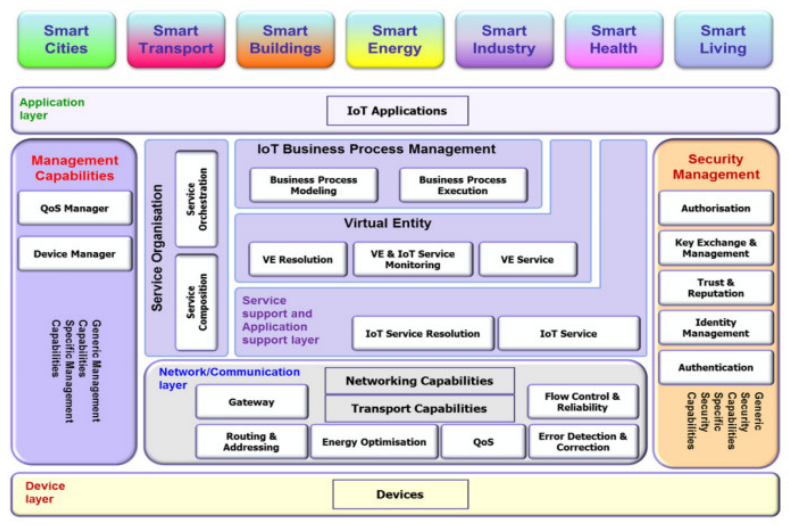

Figure 5 : Detaiiled IOT Layered Architecture( Source IERC).

\subsection{Managing security, privacy, and privacy with the IoT}

Security is one of the major problems of the internet in general, and Internet of the Objects in particular. More than $70 \%$ of connected objects are vulnerable to attacks according to the VDC analysis firm. The EagleEye program, developed by Dan Tentler, would take control of nearly one million webcams via the Showdan connected object engine. The security issue revolves around a few central issues, including the protection of personal and confidential data from intrusions of any kind (unauthorized reading, falsification, identity theft, espionage). There is also the protection of the routing channels of information against passive attacks (listening to the network), protection of the integrity of the sensors. Attention must therefore be paid to the protection of connected peripherals against the various forms of piracy: diversion of sensors, access to video surveillance data and other confidential data, authentication. To manage all these problems, protocols, algorithms and architectures have been proposed, among which APHA (Aggregate-Proof based Hierarchical Authentication scheme) for the secure transmission of data in ubiquitous and layered networks [15]. We also cite the algorithms

Cryptographic applications using arithmetic operations that offer relatively light architectures in terms of computation and resources, adapted to the environment of the embedded and the loT [17]. Other protocols offer

On the structure of the web resources by coupling information related to the context of the object to the usual identification information to solve the problem of permissions and decentralized access control to the resources and information published by the objects in the Cloud. ].

\subsection{Marking and detection technologies in IoT}

Several techniques are used in tagging and identification in the loT. DNA markers (nitrogen base chain: guanine, cytosine, thynine, adenine) use a mixture of products (liquid or powder) to develop a unique identification and authentication code for the labeled product, typically petroleum products And pharmaceutical, and technical parts of the industry. Based on synthetic DNA, DNA markers are theoretically impossible to counterfeit, given the number of combinations on the base segments (GC-T-A) [33]. RFID allows, using an identifier contained in a chip, to address many more objects than bar codes 
M. El Harrak Hajri, A Asselman, M. Yassin Chkouri. Conception and Evaluation of Internet of Things Connecting to RFID Framework. Transactions on Machine Learning and Artificial Intelligence, Vol 5 No August (2017); p: 572-583

and OCR (Optical Character Recognition) codes. RFID tags are now widely embedded in sanitary objects (containers, wagons, pallets, cartons, trolleys, plastic containers, garments) for traceability, real-time tracking and product flow coordination. The 3D digitization of the manufacturing area of the product makes it possible to put in place a so-called hybrid code which makes it possible to identify the object in a unique way. Other techniques such as the matrix code (Datamatrix, QR code, Maxi code) [34] are used for the sorting of packages in the transport of mail. Marking techniques such as

Digital code, nano-plotters (electroluminescent, nano-particles), biometrics (facial recognition, fingerprints, voices), holograms, are also used for the identification, traceability and coordination of sanitary objects.

\subsection{Performance evaluation in IoT}

Measuring the performance of a system in the loT still poses many problems because the objects must be tested under real conditions of use. Another difficulty is due to the fact that the systems in the loT are mostly based on sensors / actuators and require a more or less strong interaction with human operators. They can integrate multiple technologies and disciplines, making it much more complex to test and evaluate the performance of such systems. There are several test solutions in different degrees of realism, classified according to their architecture (2-Thirds or 3-Thirds) and the domains covered as illustrated in Figure 5. The test benches can propose generic functionalities (MoteLab, WISBED, SenseLab) or application-specific (CitySense, Friedrichshafen, Oulu Smart City). Depending on their architectures, evaluation environments can be categorized into two categories, depending on whether the architecture is two-thirds (Sensor-Server) or three-thirds (Sensor-Server-Internet). For 2-tier architectures we can mention MIRAGE, Vinelab, City Sense, FrONTS, whose limit is their inability to support the network layer. 3-tier architectures such as TWIST, INDRIYA, take into account the network layer to facilitate communication between objects and test servers, offering more flexibility and performance gains. These test solutions can integrate one or more operating systems generally used in the embedded domain (TinyOS, iSense, MoteRunner, Contiki, Sunspot) [15].

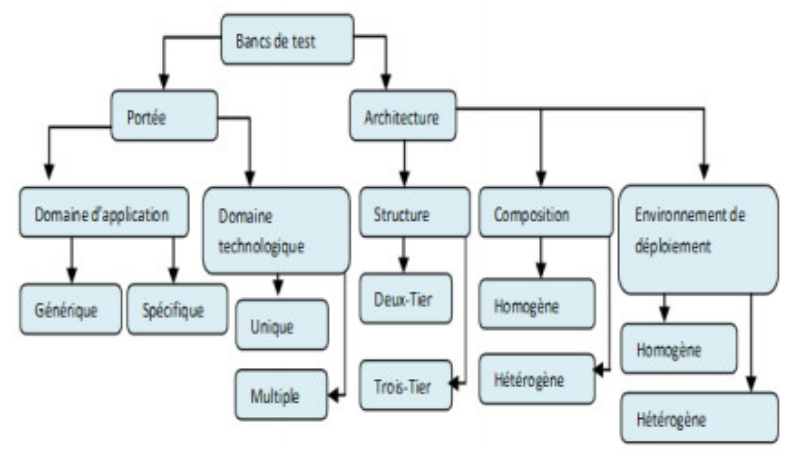

Figure 6: Classification of Assessment Technologies TheloT

\section{Conclusion}

The construction of trust goes through multiple paths. The governance of the Internet Future must be multipolar and multi-actor. The current governance of the Internet is a tool.In the context of the loT, it will have to evolve to apply to an environment.The benchmarks of private life, territorial sovereignty or 
time and memory are themselves in a phase of transformation. Regulatory measures should beAnd to ensure shared reordering of values.

To "think" internet governance in political terms th ideal-type of neutrality of theTechnique is necessary, but not sufficient. The digital economy and politicsInsidious manner of powerful constraints and, at the same time, mobility and connectionOpen up unprecedented possibilities for the democratic functioning of societies andFor better shared access to knowledge. Well-informed of these paradoxes, the institutionsAt national, European and international levels, Research in the humanities and social sciences, as well asResearch and development efforts. At the end of this study, we can only note the need for further research.Without answering all the questions posed by the Internet of the Objects, we mainlyRaised new ways of posing problems whose solutions are far from beingEvident: privacy, governance, standardization, cooperation, etc.

Each of these subjects deserves to be treated as such, and in a coordinated way, in thePerspective of the development of the Internet of Things. In addition, the need for reflection in thisSector seems urgent, both the development of technical solutions and newApplications is accelerating every day.In these circumstances, it is likely that a research effort should beTo address technical, economic, social and legal issues simultaneously.It is a prerequisite for a better understanding of the Internet ofAnd the ability of Europe to defend the values of its citizens in the new environmentdigital.

\section{REFERENCES}

[1] S. Bandyopadhyay, M. Sengupta, S. Maiti, and S. Dutta, "Role of Middleware for Internet Of Things," International Journal of Computer Science \& Engineering Survey (IJCSES), vol. 2, no. 3, pp. 94-105, 2011.

[2] M. A. Chaqfeh, N. Mohamed, P. O. Box, and A. Ain, "Challenges in Middleware Solutions for the Internet of Things," International conference on Collaboration Technologies and Systems (CTS), no. IEEE, pp. 21-26, 2012.

[3] F. Schreiber, M. Camplani, Romolo Fortunato, M. Marelli, and G. Rota, "Perla: A language and middleware architecture for data management and integration in pervasive information systems," Software Engineering, IEEE Transactions on, vol. 38, pp. 478-496, 2012.

[4] K. Henricksen, J. Indulska, and S. McFadden, Ted Balasubramaniam, "Middleware for Distributed Context-Aware Systems," in On the Move to Meaningful Internet Systems 2005: CoopIS, DOA, and ODBASE, R. Meersman and Z. Tari, Eds. Springer Berlin Heidelberg, 2005, pp. 846-863.

[5] E. Guinard, Dominique and Trifa, Vlad and Mattern, Friedemann and Wilde, "5 From the Internet of Things to the Web of Things: Resource Oriented Architecture and Best Practices," in Architecting the Internet of Things, F. Uckelmann, Dieter and Harrison, Mark and Michahelles, Ed. Springer Berlin Heidelberg, 2011, pp. 97-129.

[6] J. Sen Debasis Bandyopadhyay, "Internet of Things - Applications and Challenges in Technology and Standardization," Wireless Personal Communications, vol. 58, no. 1, pp. 49-69, 2011. 
M. El Harrak Hajri, A Asselman, M. Yassin Chkouri. Conception and Evaluation of Internet of Things Connecting to RFID Framework. Transactions on Machine Learning and Artificial Intelligence, Vol 5 No August (2017); p: 572-583

[7] ITU-T Study Group, "New ITU standards define the Internet of Things and provide the blueprints for its development," ITU, 2012. [Online]. Available: http://www.itu.int/ITU- 74 $\mathrm{T} /$ newslog/New+ITU+Standards+Define+The+Internet+Of+Things+And+Provide+The+Blu eprints+For+Its+Development.aspx.

[8] A. Bassi, "Introduction (IoT-A)," IoT-A, 2013. [Online]. Available: http://www.iota.eu/public.

[9] T. Teixeira, S. Hachem, and N. Georgantas, "Service Oriented Middleware for the Internet of Things: A Perspective)," in Towards a Service-Based Internet, vol. 257178, no. 257178, J. Abramowicz, Witold and Llorente, IgnacioM. and Surridge, Mike and Zisman, Andrea and Vayssière, Ed. Springer Berlin Heidelberg, 2011, pp. 220-229.

[10] J. Soldatos, N. Kefalakis, and N. Leontiadis, "ASPIRE Programmable Engine (APE)," ASPIRE Consortium, vol. 3, 2011.

[11] A. Salehi, M. Riahi, S. Michel, and K. Aberer, "GSN, middleware for stream world," in 10th international conference on mobile data management (MDM 2009), May, 2009, pp. 18- 20.

[12] P. Eisenhauer, Markus Rosengren and P. Antolin, Hydra: A development platform for integrating wireless devices and sensors into ambient intelligence systems. Springer, 2010, pp. 367-373. 75

[13] J. Domingues, A. Damaso, R. Nascimento, and N. Rosa, "An Energy-Aware Middleware for Integrating Wireless Sensor Networks and the Internet," International Journal of Distributed Sensor Networks, vol. 2011, pp. 1-19, 2011. [30] C. S. M. Villa, D. Rotondi, M. Comolli, (TXT), A. M. H. (Siemens) H.-P. Huth, C. K. (City), H. T. (inIT), and J. C. (EMIC), "WP 1 - PLUG\&WORK IOT REQUIREMENT ASSESSMENT AND ARCHITECTURE," 2010.

[14] D. Yazar and A. Dunkels, "Efficient application integration in IP-based sensor networks," in Proceedings of the First ACM Workshop on Embedded Sensing Systems for EnergyEfficiency in Buildings, Berkeley, California: ACM, 2009, pp. 43-48.

[15] D. Guinard, I. Ion, and S. Mayer, "In Search of an Internet of Things Service Architecture : REST or WS- *? A Developers ' Perspective," in Mobile and Ubiquitous Systems: Computing, Networking, and Services, Springer, 2012, pp. 326-337.

[16] Z. Song, A. C. Alvaro, and R. Masuoka, "Semantic Middleware for the Internet of Things," 2010. [34] L. Atzori, A. lera, and G. Morabito, "The internet of things: A survey," Computer Networks, vol. 54, no. elsevier, pp. 2787-2805, 2010.

[17] K. Paridel, E. Bainomugisha, Y. Vanrompay, Y. Berbers, and W. .Meuter, "Middleware for the internet of things, design goals and challenges," CAMPUS 2010, vol. 28, no.Electronic Communications of the EASST, pp. 1-7, 2010.

[18] Sh.zarghami, "Architectural support for dynamic homecare service provisioning," 2013. 
Transactions on Machine Learning and Artificial Intelligence Vol 5 No 4, Aug 2017

[19] Mohammad Zarifi Eslami, "Service Tailoring: A Method and Tool for User-centric Creation of Integrated IT-based Homecare Services to Support Independent Living of Elderly," Twente, 2013.

[20] A. H. Maya Daneva, Luigi Buglione, "Software Architects' Experiences of Quality Requirements: What We Know and What We Do Not Know?," Proceedings of REFSQ, pp. 1-17, 2013.

[21] R. S. Peter B. Seddon, "Towards the improved treatment of generalization of knowledge claims in IS research: drawing general conclusions from samples," EJIS 21(1), pp. 6-21, 2012 